\title{
Tendências Genéticas dos Efeitos Genéticos Direto e Materno em Características Reprodutivas de Suínos ${ }^{1}$
}

\author{
Aldrin Vieira Pires 2,5 , Paulo Sávio Lopes 3,5 , Robledo de Almeida Torres ${ }^{3,5}$, Ricardo Frederico \\ Euclydes $^{3}$, Martinho de Almeida e Silva ${ }^{4,5}$, André Ribeiro Corrêa da Costa ${ }^{2,5}$
}

\begin{abstract}
RESUMO - Foram utilizados dados de peso da leitegada ao nascimento (PLN), peso da leitegada aos 21 dias (PL21), tamanho de leitegada ao nascimento (TLN), tamanho de leitegada ao desmame (TLD) e taxa de mortalidade (TM), para avaliar a tendência genética atribuída aos efeitos genéticos aditivos diretos e maternos, em suínos Duroc, Landrace e Large White. As estimativas dos componentes de (co)variância foram obtidas pelo método da máxima verossimilhança restrita (REML). As tendências genéticas dos efeitos genéticos direto e materno foram calculadas pela regressão das médias dos valores genéticos preditos das características, em relação ao ano de nascimento das porcas. As estimativas de tendências genéticas dos efeitos diretos mostraram que pouco ou praticamente nenhum progresso ocorreu nas características de leitegada, havendo tendências genéticas negativas (-0,0382 a 0,0756 kg no PLN, -0,1119 a 0,1118 kg no PL21, -0,0031 a 0,0509 leitões no TLN, -0,0217 a 0,0084 leitões no TLD e 0,0997 a -0,0059\% na TM), evidenciando a dificuldade de se obterem ganhos genéticos expressivos nas características reprodutivas. Estes resultados destacam a importância de se utilizar a informação de parentes no melhoramento genético destas características, para otimizar os ganhos por seleção. As estimativas de tendência genética dos efeitos genéticos materno apresentaram-se, em geral, negativas, possivelmente em função das correlações genéticas negativas entre os efeitos genéticos aditivos direto e materno.
\end{abstract}

Palavras-chave: BLUP, características reprodutivas, suínos, tendências genéticas

\section{Genetic Trends for Maternal and Direct Genetic Effects on Reproductive Traits of Swine}

\begin{abstract}
Data of Duroc, Landrace and Large White swine were used to estimate genetic trends for maternal and direct genetic effects of litter size (TLN) and litter weight (PLN) born alive, litter size (TLD) and litter weight (PL21) at 21 days and mortality rate (TM). The genetic parameters were estimated by restricted maximum likelihood (REML) method. Estimated genetic direct and maternal trends were obtained by regressing genetic values averages on dam birth year. Estimated genetic direct trends showed no progress or very small progress for litter traits, being these progresses, in some cases, even negatives (-0.0382 to $0.0756 \mathrm{~kg}$ in PLN, -0.1119 to 0.1118 $\mathrm{kg}$ in PL21, -0.0031 to 0.0509 pigs in TLN, -0.0217 to 0.0084 pigs in TLD and 0.0997 to $-0.0059 \%$ in TM), showing the difficulty to get high genetic gain in reproductive traits. These results showed the importance of considering relationships informations, in order to obtain higher selection gain for these traits. Estimated genetic maternal trends were, in general, negatives, possibly in function of negative genetic correlations among direct and maternal genetic additive effect.
\end{abstract}

Key Words: BLUP, genetic trends, reproductive traits, swine

\section{Introdução}

Com a finalidade de se obter um produto de qualidade, em grande quantidade, e com mínimo custo, várias inovações tecnológicas vêm sendo desenvolvidas na suinocultura. Para se obter um suíno terminado de boa qualidade, torna-se necessário que o leitão seja desmamado em boas condições, visto que o desempenho dos animais em crescimento e terminação é dependente de seu desenvolvimento nas fases anteriores: gestação, aleitamento e creche.
Verifica-se, então, que a performance reprodutiva, em especial o tamanho e o peso de leitegada, é essencial para o sucesso da criação de suínos. Alguns melhoristas, cientes da importância destas características, passaram a incluí-las no processo de avaliação genética.

O estudo das tendências genéticas permite avaliar a mudança ocasionada pelo processo de seleção, em determinada característica, ao longo dos anos.

As alterações produzidas pela seleção na composição genética de um rebanho são difíceis de serem

\footnotetext{
1 Parte da Tese do primeiro autor para a obtenção do título de Mestre em Zootecnia - UFV.

2 MS em Zootecnia, DZO, UFV, Viçosa, MG, CEP: 36571-000.E-mail: avpires@alunos.ufv.br

3 Professor do DZO, UFV, Viçosa, MG - CEP: 36571-000.

4 Professor da UENF, Campos, RJ.
} 
quantificadas. Entretanto, alguns métodos permitem a identificação de causas responsáveis pelas mudanças na média da população, como resultado de variações no mérito genético e das condições de ambiente (CHENETTE et al., 1982).

É interessante observar que parece existir correlação negativa entre as mudanças genéticas e de ambiente, tendo em vista que, em contraposição à alta mudança genética, existe mudança ambiental negativa (Van VLECK, 1982).

A utilização de grupos controles e a adoção de esquema de seleção divergente são procedimentos propostos por HILL (1972a, b) para remover a influência ambiental. Porém, estes dois procedimentos são muito difíceis de serem estabelecidos, principalmente em razão do custo de se manterem populações controle.

SORENSEN e KENNEDY (1984), usando dados simulados, demonstraram que, sob determinadas condições, as equações de modelos mistos dispensam o uso de populações controle como meio para decompor adequadamente a tendência fenotípica em seus componentes genéticos e ambientais. Segundo os autores, se as variâncias das características antes da seleção forem conhecidas, a seleção for uma função linear dos dados e a matriz de parentesco for completa, a tendência genética pode ser estimada por meio da metodologia de modelos mistos.

Sabe-se que, nos mamíferos, as mães exercem efeito maior que os pais sobre o fenótipo dos descendentes, pois, além da contribuição genética, podem influenciar a progênie por meio do ambiente que lhe proporciona. Assim, as características de crescimento, principalmente até o desmame, são determinadas por dois genótipos: o do próprio animal (efeito genético direto) e o de sua mãe (efeito genético materno). Entretanto, alguns autores verificaram efeito materno significativo em características que se expressam mais tarde na vida do animal (BRYNER et al., 1992; POLASTRE et al., 1992; LEDUR et al., 1994; e PIRES, 1999).

Diversos autores têm encontrado correlações genéticas negativas entre efeito direto e materno para várias características e raças. Portanto, tem-se sugerido a inclusão do efeito materno nos modelos para estimação de parâmetros genéticos (NOBRE et al., 1988; POLASTRE et al., 1992; SOUSA et al., 1995; COBUCCI et al., 1997; PIRES, 1999, entre outros) e para avaliação genética (MATHERSON et al., 1974; NOBRE et al., 1988; ELER et al., 1989; DE
LOS REYES et al., 1994; PIRES, 1999, entre outros).

Este trabalho foi conduzido com o objetivo de avaliar as tendências genéticas dos efeitos diretos e maternos de características de leitegada em suínos.

\section{Material e Métodos}

Os dados utilizados neste trabalho são provenientes de animais da empresa COOPERCENTRAL, situada no município de Chapecó-SC, das raças Duroc, Landrace e Large White.

Ao nascimento, os leitões foram submetidos ao seguinte manejo: corte dos dentes, mossagem, pesagem e aplicação de ferro dextrano. Foi também realizada a padronização das leitegadas, sendo as transferências feitas somente dentro das raças. A partir do décimo dia até uma semana após o desmame, os leitões receberam ração pré-inicial. A maioria dos animais foi pesada aos 21 dias de idade. Os animais que por algum motivo não foram pesados aos 21 dias tiveram seus pesos ajustados para esta idade, de acordo com a seguinte fórmula:

$$
\mathrm{PAD}=([\{\mathrm{POD}-1,4\} / \mathrm{ID}] * 21)+1,4
$$

em que PAD é peso ajustado do leitão para 21 dias; POD, peso observado do leitão na pesagem a, aproximadamente, 21 dias; ID, idade do leitão no dia da pesagem; e 1,4, peso padrão adotado para o peso ao nascer.

O desmame foi feito entre 24 e 28 dias de idade, não sendo feita a pesagem ao desmame. PIRES (1999) apresenta maiores detalhes acerca do manejo dos animais.

As características estudadas foram: tamanho da leitegada ao nascer (TLN), tamanho de leitegada ao desmame (TLD), peso da leitegada ao nascer (PLN), peso da leitegada corrigido para os 21 dias de idade (PL21) e taxa de mortalidade do nascimento ao desmame (TM).

O efeito fixo de grupo contemporâneo (GC) e ordem de parição como covariável foi considerado nos modelos, sendo que para as raças Landrace e Large White GC foi constituído por rebanho, ano e estação de parição e, para a raça Duroc GC, apenas pelos efeitos de ano e pela estação de parição.

As estimativas dos componentes de (co)variância foram obtidas pelo REML, utilizando o programa MTDFREML (BOLDMAN et al., 1995). Estes componentes foram utilizados para predizer os valores genéticos de cada raça, pelo BLUP.

Foi adotado o seguinte modelo: 
Rev. bras. zootec.

$$
\mathrm{y}=\mathrm{X} \underset{\sim}{\mathrm{b}}+\mathrm{Z}_{1} \underset{\sim}{\mathrm{d}}+\mathrm{Z}_{2} \underset{\sim}{\mathrm{m}}+\mathrm{Z}_{3} \mathrm{p}+\underset{\sim}{\mathrm{e}}
$$

em que $y$, vetor de observações; $X$, matriz de incidência de efeitos fixos; $b$, vetor de efeitos fixos; $Z_{1}$, matriz de incidência de efeitos genéticos aditivos diretos; $\mathrm{d}_{\sim}$, vetor de efeitos genéticos aditivos diretos; $\mathrm{Z}_{2}$, matriz de incidência de efeitos genéticos aditivos maternos; $\mathrm{m}_{\sim}$, vetor de efeitos genéticos aditivos maternos; $Z_{3}$, matriz de incidência de efeitos comuns de leitegada; $p$, vetor de efeitos comuns de leitegada; $\stackrel{\mathrm{e}}{\sim}$, vetor de efeito residual.

Foi utilizado como critério de convergência a variância dos valores do simplex $\left(-2 \log _{e}\right.$ de verossimilhança) inferior a $10^{-9}$. Após cada convergência, o programa foi reiniciado, usando as estimativas obtidas anteriormente como valores iniciais. Este procedimento então foi repetido até que as diferenças entre as estimativas, das duas últimas convergências, fossem menores que $10^{-5}$.

As tendências genéticas dos efeitos direto e materno foram calculadas pela regressão das médias dos valores genéticos preditos em relação ao ano de nascimento das porcas. Os cálculos da regressão linear foram efetuados utilizando o software SAEG 7.1 (EUCLYDES, 1997).

\section{Resultados e Discussão}

As médias e os desvios-padrão das características para cada raça são apresentados na Tabela 1 .

Os gráficos das tendências genéticas dos efeitos genéticos diretos estão apresentados nas Figuras 1, 2, 3,4 e 5 . As estimativas das tendências genéticas

Tabela 1 - Número de suínos, médias e desvios-padrão das características estudadas Table 1 - Number of swines, averages and standard errors of traits in study

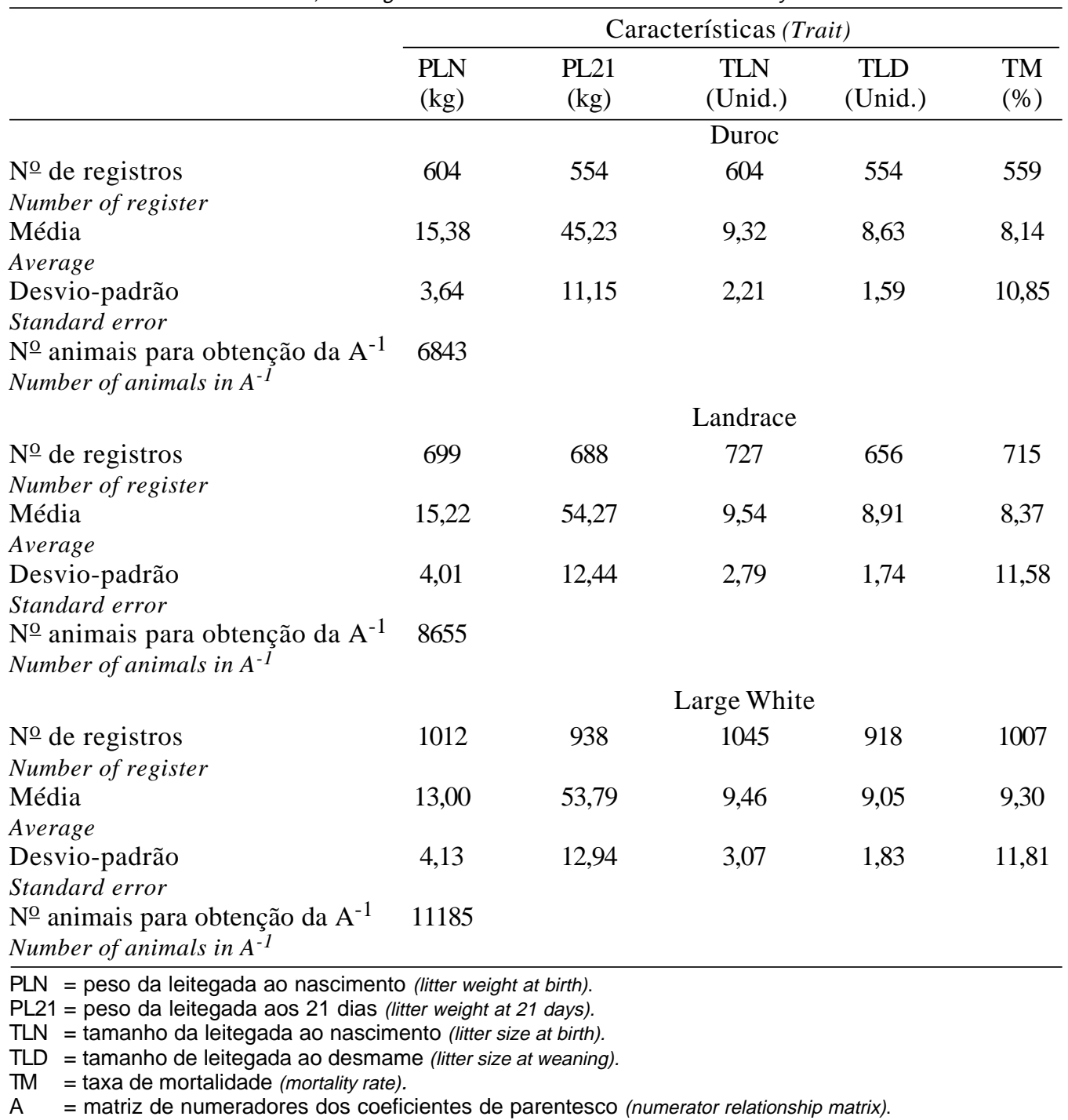




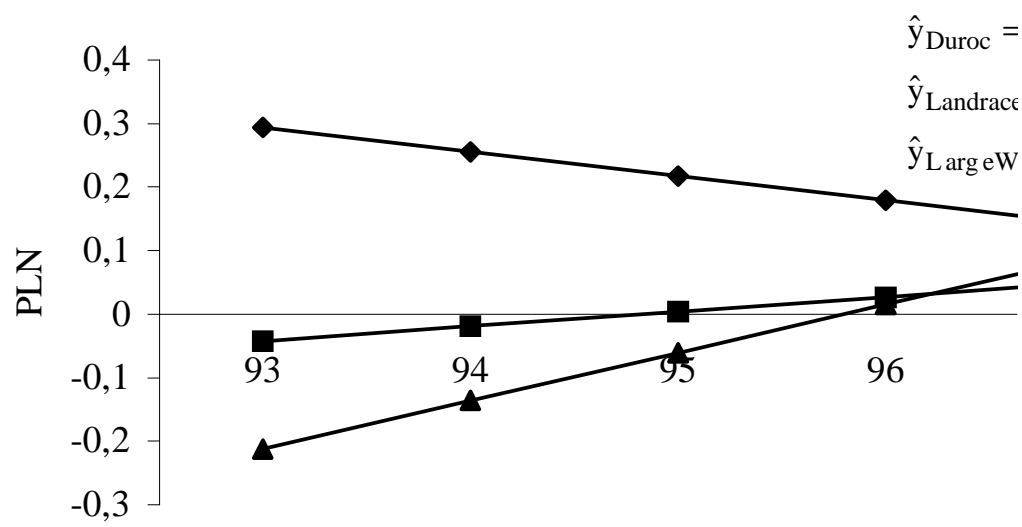

Ano

Figura 1 - Tendências genéticas dos efeitos genéticos diretos para peso da leitegada ao nascimento (PLN), em $\mathrm{kg}$, para as raças Duroc, Landrace e Large White, no período de 1993 a 1997.

Figure 1 - Direct genetic trends for litter weight at birth (PLN), in kg, for Duroc, Landrace and Large White breeds, from 1993 to 1997.

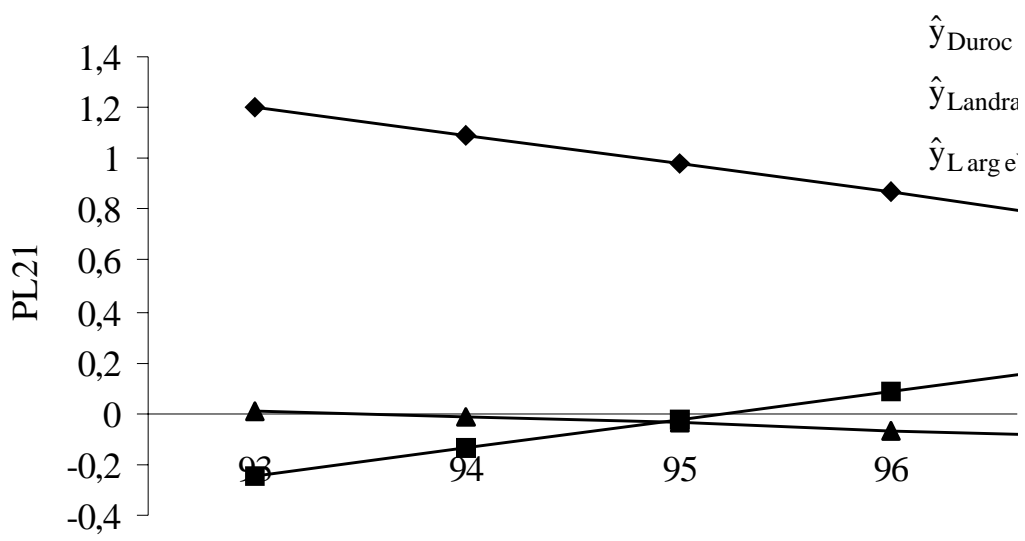

Ano

Figura 2 - Tendências genéticas dos efeitos genéticos diretos para peso da leitegada ao desmame, ajustado para 21 dias (PL21) em kg, para as raças Duroc, Landrace e Large White, no período de 1993 a 1997.

Figure 2 - Direct genetic trends for litter weight at weaning, in kg, adjusted to 21 days (PL21) in kg, for Duroc, Landrace and Large White breeds, from 1993 to 1997.

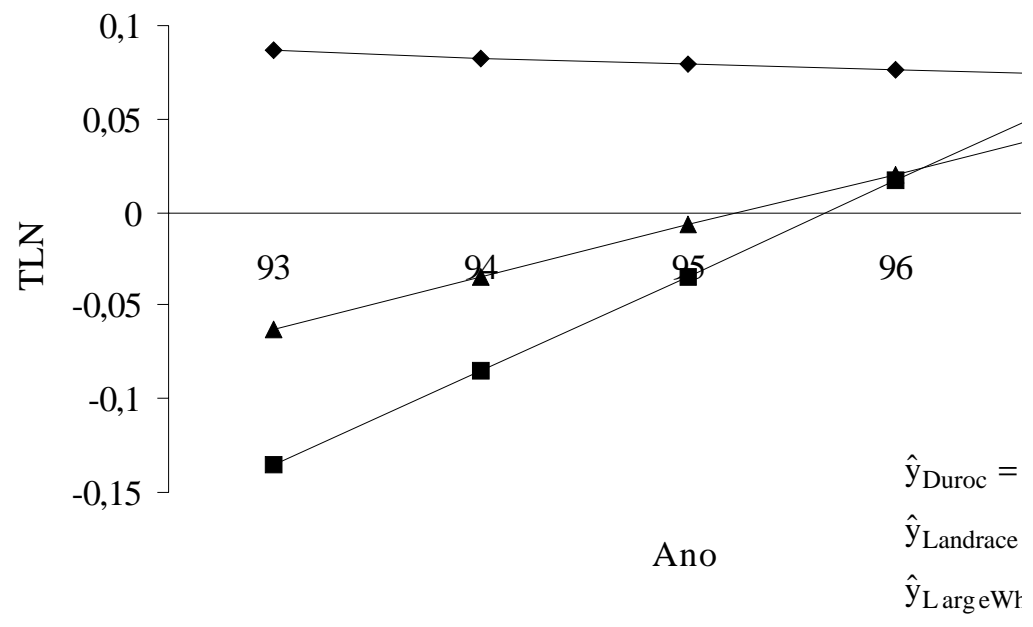

Figura - Tendências genéticas dos efeitos genéticos diretos para tamanho de leitegada ao nascimento (TLN) em no de leitões, para as raças Duroc, Landrace e Large White, no período de 1993 a 1997.

Figure 3 - Direct genetic trends for litter size at birth (TLN), in number of pigs, for Duroc, Landrace and Large White breeds, from 1993 to 1997. 
Rev. bras. zootec.

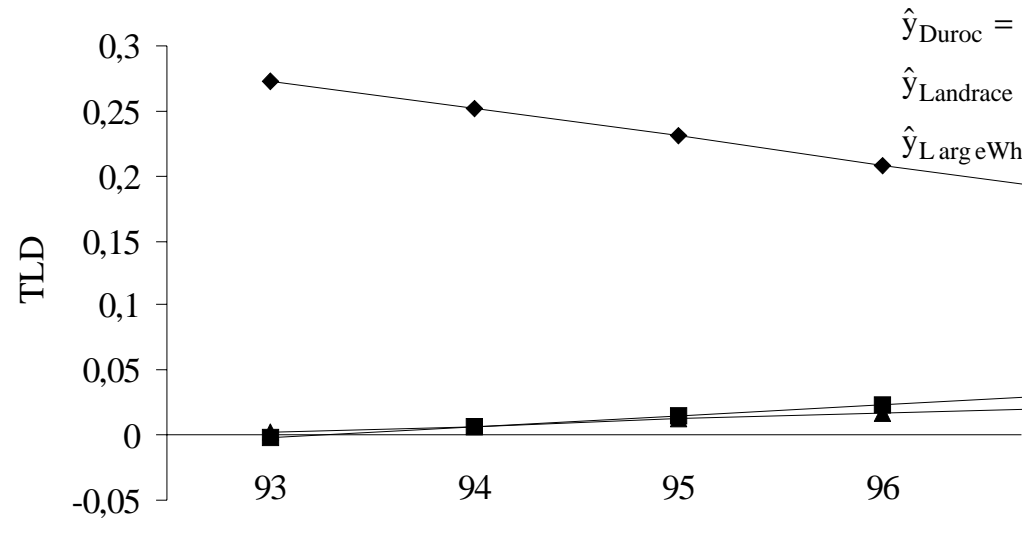

Ano

Figura 4 - Tendências genéticas dos efeitos genéticos diretos para tamanho de leitegada ao desmame (TLD), em no de leitões, para as raças Duroc, Landrace e Large White, no período de 1993 a 1997.

Figure 4 - Direct genetic trends for litter size at weaning (TLD), in number of pigs, for Duroc, Landrace and Large White breeds, from 1993 to 1997.

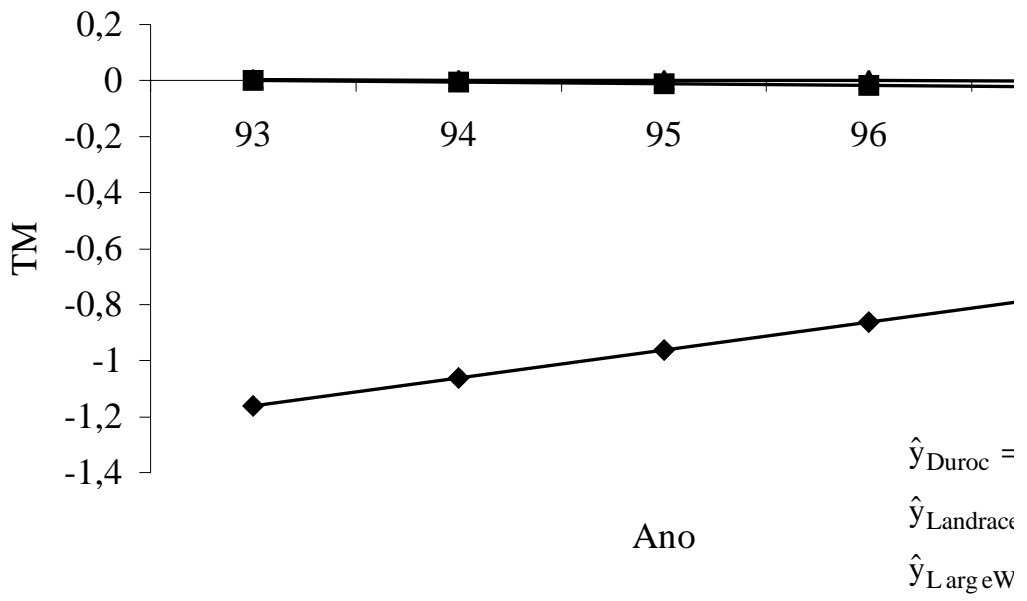

Figura 5 - Tendências genéticas dos efeitos genéticos diretos para taxa de mortalidade (TM) em percentagem, para as raças Duroc, Landrace e Large White, no período de 1993 a 1997.

Figure 5 - Direct genetic trends for mortality rate (TM), in percentage, for Duroc, Landrace and Large White breeds, from 1993 to 1997.

anuais, referentes ao efeito genético direto, com os respectivos desvios-padrão, para as características peso da leitegada ao nascimento (PLN), peso da leitegada aos 21 dias (PL21), tamanho de leitegada ao nascimento (TLN), tamanho de leitegada ao desmame (TLD) e taxa de mortalidade (TM), nas três raças, encontram-se na Tabela 2.

Observaram-se, em alguns casos, baixos coeficientes de determinação $\left(\mathrm{r}^{2}\right)$, indicando que não houve tendências genéticas definidas.

As raças Landrace e Large White tiveram comportamento similar nas tendências dos efeitos genéticos direto, sendo que ambas apresentam tendências distintas da raça Duroc, o que pode ser evidenciado nas Figuras de 1 a 5. Isso se deve ao fato de as raças Landrace e Large White produzirem matrizes híbridas destinadas aos rebanhos comerciais, ou seja, devido ao fato de serem linhas maternas.

No entanto, o progresso genético ao longo dos anos, nas características PLN, PL21, TLN, TLD e $\mathrm{TM}$, foi muito pequeno, sugerindo pouca ou nenhuma atenção dada a estas características no programa de melhoramento genético. Deve ser acrescentado ainda o fato de que tais características apresentam baixa herdabilidade, o que dificulta ainda mais o progresso genético. IRGANG et al. (1997) também encontraram ganho genético pouco expressivo em número de 
Tabela 2 - Estimativas das tendências genéticas anuais e respectivos desvios-padrão das características, para o efeito genético direto

Table 2 - Annual genetic trends estimates and respective traits standard errors, for direct genetic effect

\begin{tabular}{lccr}
\hline & \multicolumn{3}{c}{ Raça (Breed) } \\
\cline { 2 - 4 } $\begin{array}{l}\text { Características } \\
\text { Trait }\end{array}$ & Duroc & Landrace & Large White \\
\hline PLN $(\mathrm{kg})$ & $-0,0382 \pm 0,0137$ & $0,0232 \pm 0,0103$ & $0,0756 \pm 0,0276$ \\
PL21 $(\mathrm{kg})$ & $-0,1119 \pm 0,0434$ & $0,1118 \pm 0,0931$ & $-0,0249 \pm 0,0235$ \\
TLN & $-0,0031 \pm 0,0069$ & $0,0509 \pm 0,0199$ & $0,0279 \pm 0,0121$ \\
TLD & $-0,0217 \pm 0,0115$ & $0,0084 \pm 0,0124$ & $0,0049 \pm 0,0034$ \\
TM $(\%)$ & $0,0997 \pm 0,0339$ & $-0,0059 \pm 0,0102$ & $-0,0014 \pm 0,0006$ \\
\hline
\end{tabular}

PLN = peso da leitegada ao nascimento (litter weight at birth).

PL21 = peso da leitegada aos 21 dias (litter weight at 21 days).

TLN = tamanho da leitegada ao nascimento (litter size at birth).

TLD = tamanho de leitegada ao desmame (litter size at weaning).

$\mathrm{TM}=$ taxa de mortalidade (mortality rate)

leitões nascidos vivos nas raças Landrace e Large White.

Percebe-se na raça Duroc que as tendências genéticas para PLN, PL21, TLN e TLD foram negativas. Isso ocorreu em função da correlação genética negativa entre estas características reprodutivas e as de desempenho. Uma vez que os programas de melhoramento desta raça têm o objetivo de produzir machos (raça paterna), as características de desempenho são prioritárias, determinando, assim, resposta correlacionada negativa nas características reprodutivas.

No processo de avaliação de suínos, sobretudo das raças Landrace e Large White, as características reprodutivas, tamanho e peso da leitegada ao nascimento e ao desmame, juntamente com taxa de mortalidade, devem ser incluídas como critério de seleção e, ou, descarte, para que assim se obtenham maiores ganhos, e conseqüente aumento na produtividade.
O uso de ferramentas sofisticadas de avaliação genética, como o BLUP e modelo animal, deve resultar em melhores predições de valor genético (SOUTHWOOD e KENNEDY, 1991) e seleção mais acurada de animais hiperprolíficos (BIDANEL et al., 1994), aumentando as possibilidades de obtenção de ganhos genéticos no tamanho de leitegada de suínos.

Os gráficos das tendências genéticas dos efeitos genéticos maternos estão apresentados nas Figuras $6,7,8,9$ e 10. As estimativas das tendências genéticas anuais com os respectivos desvios-padrão, para as características PLN, PL21, TLN, TLD e TM, nas três raças, são apresentadas na Tabela 3 .

As tendências genéticas dos efeitos genéticos maternos apresentaram-se negativas ou praticamente nulas, indicando inexistência de preocupação com tal efeito nos programas de melhoramento das populações estudadas.

Tabela 3 - Estimativas das tendências genéticas anuais e respectivos desvios-padrão das características, nas três raças, para o efeito genético materno

Table 3 - Annual genetic trends estimates and respective traits standard errors, for maternal genetic effect

\begin{tabular}{lccr}
\hline & & Raça $($ Breed $)$ \\
Características & Duroc & Landrace & Large White \\
\cline { 2 - 4 } Trait & & & \\
\hline PLN $(\mathrm{kg})$ & $-0,0272 \pm 0,0094$ & $-0,0457 \pm 0,0203$ & $0,0238 \pm 0,0086$ \\
PL21 $(\mathrm{kg})$ & $-0,0059 \pm 0,0023$ & $-0,0164 \pm 0,0136$ & $-0,0182 \pm 0,0172$ \\
TLN & $-0,0047 \pm 0,0105$ & $-0,0070 \pm 0,0101$ & $-0,0073 \pm 0,0032$ \\
TLD & $0,0013 \pm 0,0007$ & $-0,0031 \pm 0,0060$ & $-0,0042 \pm 0,0038$ \\
TM $(\%)$ & $0,0148 \pm 0,0051$ & $-0,0098 \pm 0,0168$ & $0,0003 \pm 0,0002$ \\
\hline
\end{tabular}

PLN = peso da leitegada ao nascimento (litter weight at birth).

PL21 = peso da leitegada aos 21 dias (litter weight at 21 days).

TLN = tamanho da leitegada ao nascimento (litter size at birth).

TLD = tamanho de leitegada ao desmame (litter size at weaning).

$\mathrm{TM}=$ taxa de mortalidade (mortality rate). 
Rev. bras. zootec.

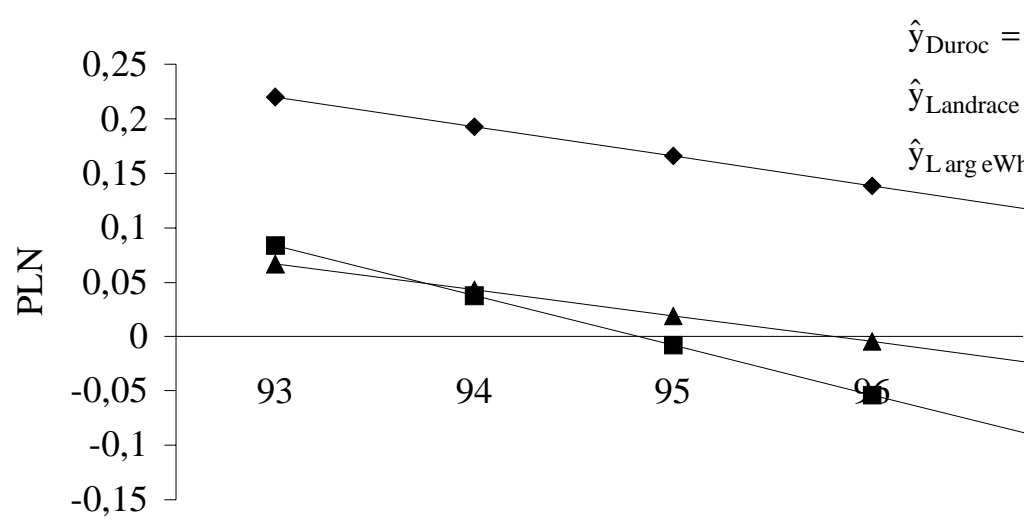

Ano

Figura 6 - Tendências genéticas dos efeitos genéticos maternos para peso da leitegada ao nascimento (PLN), em $\mathrm{kg}$, para as raças Duroc, Landrace e Large White, no período de 1993 a 1997.

Figure 6 - Maternal genetic trends for litter weight at birth (PLN), in kg, for Duroc, Landrace and Large White breeds, from 1993 to 1997.

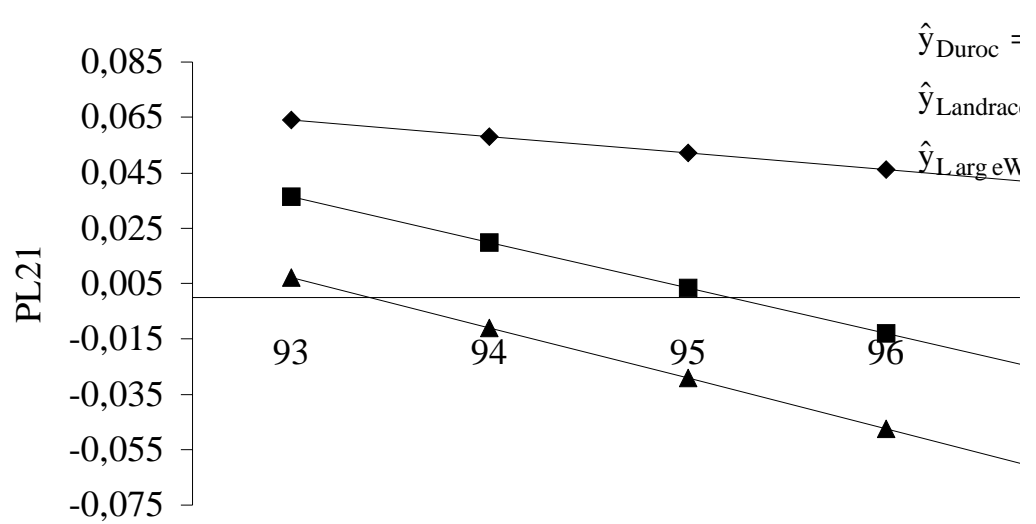

Ano

Figura 7 - Tendências genéticas dos efeitos genéticos maternos para peso da leitegada ao desmame, ajustado para 21 dias (PL21), em kg, para as raças Duroc, Landrace e Large White, no período de 1993 a 1997.

Figure 7 - Maternal genetic trends for litter weight at weaning, adjusted to21 days (PL21), in kg, for Duroc, Landrace and Large White breeds, from 1993 to 1997.

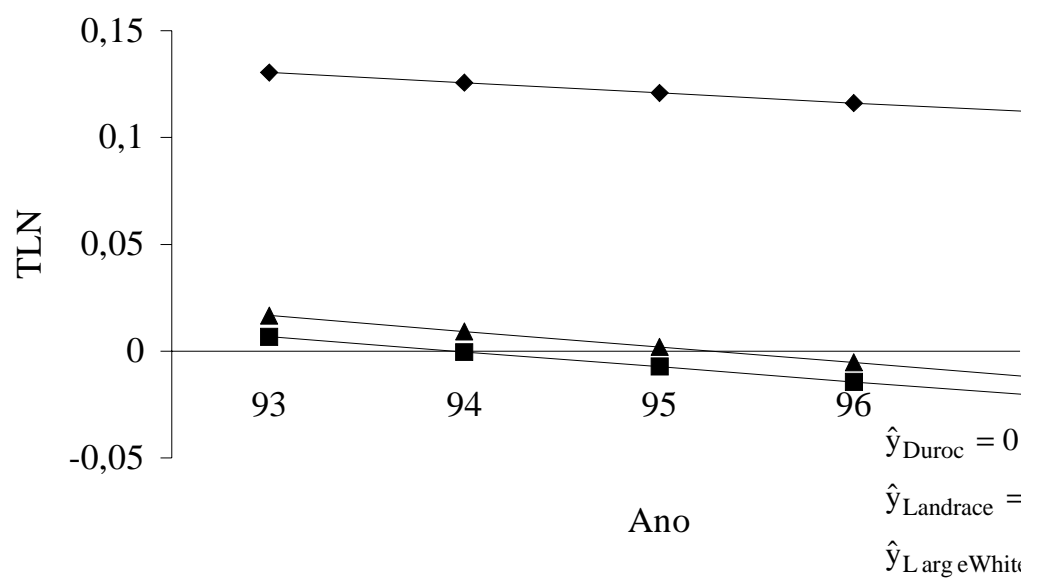

Figura 8 - Tendências genéticas dos efeitos genéticos maternos para tamanho de leitegada ao nascimento (TLN), em no de leitões, para as raças Duroc, Landrace e Large White, no período de 1993 a 1997.

Figure 8 - Maternal genetic trends for litter size at birth (TLN), in number of pigs, for Duroc, Landrace and Large White breeds, from 1993 to 1997. 
PIRES et al.

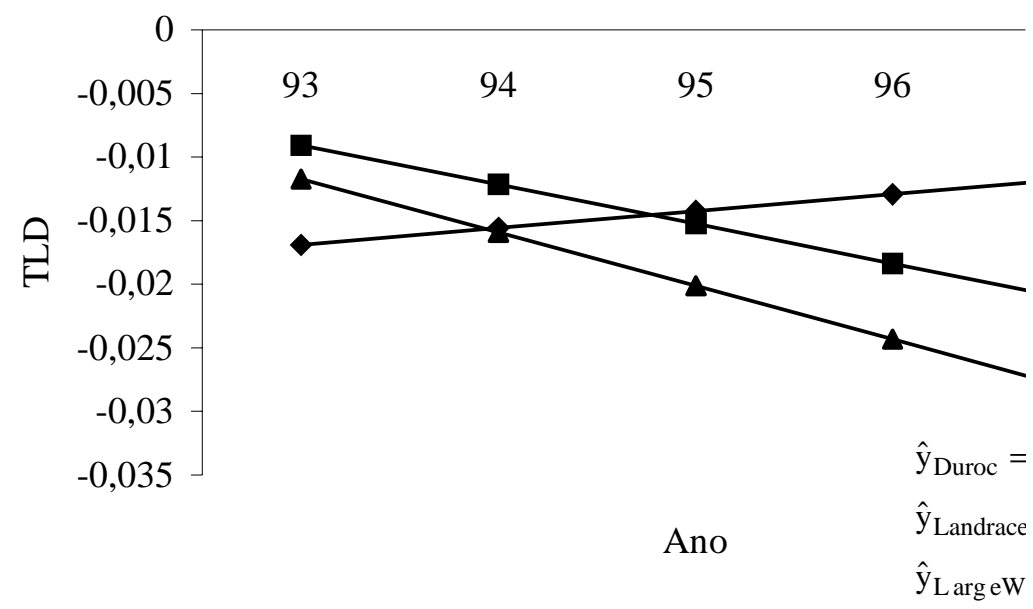

Figura 9 - Tendências genéticas dos efeitos genéticos maternos para tamanho de leitegada ao desmame (TLD), em no de leitões, para as raças Duroc, Landrace e Large White, no período de 1993 a 1997.

Figure 9 - Maternal genetic trends for litter size at weaning (TLD), in number of pigs, for Duroc, Landrace and Large White breeds, from 1993 to 1997.

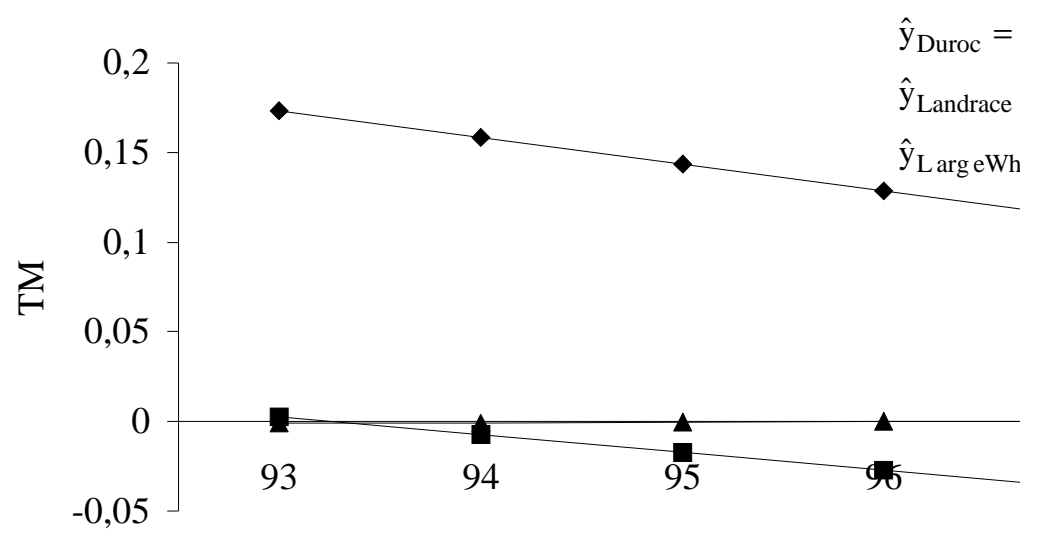

Ano

Figura 10 - Tendências genéticas dos efeitos genéticos maternos para taxa de mortalidade (TM), em percentagem, para as raças Duroc, Landrace e Large White, no período de 1993 a 1997.

Figure 10 - Maternal genetic trends for mortality rate (TM), in percentage, for Duroc, Landrace and Large White breeds, from 1993 to 1997.

Segundo ROEHE e KENNEDY (1993), o efeito genético materno influencia a reposta pela seleção direta para tamanho de leitegada ao nascimento, e as estimativas de tendências genéticas e ambientais para tamanho de leitegada são viesadas, quando o efeito materno for ignorado.

BRADFORD (1972), estudando efeito materno em cabras, citou que há a necessidade de programas genéticos destinados a melhorar o desempenho materno, não apenas por causa da contribuição direta deste componente, mas também pelo aparente efeito do ambiente materno na expressão da variação genética na taxa de crescimento. Sugere ainda maior ênfase ao desenvolvimento de linhas fêmeas especializadas com desempenho materno superior.

Os trabalhos de tendência genética materna são raros, sobretudo em suínos. Os efeitos genéticos aditivos maternos têm recebido atenção apenas do ponto de vista teórico. PIRES (1999), estudando efeito materno em características de leitegada em suínos, concluiu que a inclusão do efeito materno, juntamente com os efeitos permanentes de meio, foi significativa para a maioria das características e, portanto, deve fazer parte dos modelos, no processo de avaliação animal, principalmente no que se refere a raças utilizadas para produzir fêmeas, em que a habilidade materna em produzir com qualidade e quantidade deve merecer atenção adequada. 
Outro fator que pode ajudar a explicar estas baixas, nulas ou negativas estimativas de tendência genética dos efeitos direto e materno, é o antagonismo existente entre tais efeitos, verificado pela altas e negativas correlações entre eles. Dessa forma, ao se selecionarem indivíduos apenas com base nas informações de seus efeitos genéticos diretos, a habilidade ou capacidade materna destes será reprimida e, assim, a melhoria genética da população será prejudicada.

\section{Conclusões}

As estimativas de tendência genética dos efeitos diretos evidenciam a dificuldade de se obterem ganhos genéticos expressivos nas características reprodutivas.

As correlações genéticas negativas entre os efeitos genéticos aditivos direto e materno, são, possivelmente, responsáveis pelas estimativas negativas de tendência genética do efeito genético materno.

\section{Agradecimento}

À COOPERCENTRAL, pela cessão dos dados, o que possibilitou a execução deste trabalho.

\section{Referências Bibliográficas}

BIDANEL, J.P., GRUAND, J., LEGAULT, C. An overview of twenty years of selection for litter size in pigs using "hyperprolific" schemes. In: WORLD CONGRESS ON GENETICS APPLIED TO LIVESTOCK PRODUCTION, 5, 1994, Guelph. Proceedings... Guelph: University of Guelph, 1994. v.17, p.512-515.

BRYNER, S.M., MABRY, J.W., BERTRAND, J.K. et al. 1992. Estimation of direct e maternal heritability and genetic correlation for backfat and growth rate in swine using data from centrally tested Yorkshire boars. J. Anim. Sci., 70(6): 1755-1759.

BOLDMAN, K.G., KRIESE L.A., VAN VLECK, L.D. et al. A Manual for Use of MTDFREML. A set of programs to obtain estimates of variance and covariance [Draft]. Lincoln, U.S. Department of Agriculture, Agriculture Research Service, 1995. 115p.

BRADFORD, G.E. 1972. The role of maternal effects in animal breeding: VII.Maternal effects in sheep. J. Anim. Sci., 35(6):1324-1334.

CHENETTE, C.G., FRAHM, R.R., WHITEMAN, J.V. 1982. Direct and correlated responses to selection for increase weaning and yearling weights in Hereford cattle. II. Evaluation of response. Anim. Sci. Res. Rep., 112:301-307.

COBUCCI, J.A., OLIVEIRA, A.I.G., GONÇALVES, T.M. 1997. Parâmetros genéticos de peso ao nascer em suínos híbridos pelos métodos dos quadrados mínimos e da máxima verossimilhança. Rev. Ciência e Agrotecnologia, 21(4):511-515.

DE LOS REYES, A., LÔBO, R.B., OLIVEIRA, H.N. et al. Estimativa de efeitos genético direto e maternal para pesos até
365 dias, usando modelo animal bivariado em rebanhos Nelore. In: REUNIÃO ANUAL DA SOCIEDADE BRASILEIRA DE ZOOTECNIA, 31, 1994, Maringá. Anais.. Maringá: SBZ, 1994. p.167.

ELER, J.P., LOBO, R.B., DUARTE, F.A.M. 1989. Avaliação dos efeitos genéticos direto e materno em pesos de bovinos da raça Nelore criados no Estado de São Paulo. R. Soc. Bras. Zootec., 18(2):112-123.

EUCLYDES, R.F. 1997. Sistema para análises estatísticas (SAEG) versão 7.1. Viçosa, MG: UFV. 152p.

HILL, W.G. 1972a. Estimation of realized heritabilities from selection experiments. I. Selection in one direction. Biometrics, 28(3):767-780

HILL, W.G. 1972b. Estimation of realized heritabilities from selection experiments. II. Divergent selection. Biometrics, 28(3):747-765.

IRGANG, R., FÁVERO, J.A., MEDEIROS, G. Tendências fenotípicas e genéticas do número de leitões nascidos vivos em porcas Landrace e Large White. In: REUNIÃO ANUAL DA SOCIEDADE BRASILEIRA DE ZOOTECNIA, 34, 1997, Juiz de Fora. Anais... Juiz de Fora: SBZ, 1997. 3:320-323.

LEDUR, M.C., CARREGAL, R.D., SOBRINHO, E.B. 1994. Avaliação da heterose e da habilidade materna na fase de crescimento de coelhos de corte. R. Soc. Bras. Zootec., 23(2):165-172.

MATHERSON, G., POUJARDIEU, B. LEFORT, G. A model of estimation the genetic parameters in the presence of genetics direct and maternal effects in the rabbits. In: WORLD CONGRESS ON GENETICS APLIED TO LIVESTOCK PRODUCTION, 1974, [s.1.]. Proceedings.. 1974, v.3, p.447-453.

NOBRE, P.R.C., EUCLIDES FILHO, K., ROSA, A.N. 1988 Componentes materno e direto das tendências genéticas para pesos em gado Nelore. R. Soc. Bras. Zootec., 17(1):01-16.

PIRES, A.V. Avaliação genética de características reprodutivas em suínos. Viçosa, MG: Universidade Federal de Viçosa 1999. 83p. Dissertação (Mestrado em Zootecnia) - Universidade Federal de Viçosa,1999.

POLASTRE, R., MOURA, A.S.A.M.T., CARMELO, M.J 1992. Estudo de efeitos genéticos direto e materno em características de produção de coelhos Selecta. R. Soc. Bras. Zootec., 21(5):855-865.

ROEHE, R., KENNEDY, B.W. 1993. Efficiency of an approximate animal model for maternal and direct genetic effects of litter size in swine. J. Anim. Sci., 71(12):3251-3260.

SORENSEN, D.A., KENNEDY, B.W. 1984. Estimation of response to selection using least-squares and mixed model methodology. J. Anim. Sci., 58(5):1097-1106.

Van VLECK, L.D. 1982. Theoretical and actual genetic progress in dairy cattle. Acta Agric. Scan., 32:48-60.

SOUSA, W.H., TORRES, R.A., LEITE, P.R.M. Estimativas de componentes de variância devido aos efeitos direto e materno para características de crescimento em caprinos. In: REUNIÃO ANUAL DA SOCIEDADE BRASILEIRA DE ZOOTECNIA, 32, 1995, Brasília. Anais... Brasília: SBZ, 1995. p.721-722.

SOUTHWOOD, O.I., KENNEDY, B.W. 1991. Genetic and environmental trends for litter size in swine. J. Anim. Sci., 69(8):3177-3182.

Recebido em: 26/05/99

Aceito em: 02/05/00 